\title{
Prevalence and associated factors of anxiety and depression among patients with oromaxillofacial venous malformations
}

\author{
Xiaochen Zhang ${ }^{1,2} \wedge$, Xiaomei Zhao ${ }^{1}$, Ping Wu ${ }^{1}$, Zeliang Zhao ${ }^{1,2}$, Ming Liu ${ }^{1}$, Yan-An Wang ${ }^{1,2} \wedge$ \\ ${ }^{1}$ Department of Oral and Maxillofacial-Head and Neck Oncology, Ninth People's Hospital, School of Medicine, Shanghai Jiao Tong University, \\ Shanghai, China; ${ }^{2}$ Shanghai Key Laboratory of Stomatology \& Shanghai Research Institute of Stomatology, National Clinical Research Center of \\ Stomatology, Shanghai, China \\ Contributions: (I) Conception and design: YA Wang; (II) Administrative support: M Liu; (III) Provision of study materials or patients: X Zhao, YA \\ Wang; (IV) Collection and assembly of data: X Zhang, P Wu, Z Zhao; (V) Data analysis and interpretation: X Zhang; (VI) Manuscript writing: All \\ authors; (VII) Final approval of manuscript: All authors. \\ Correspondence to: Ming Liu; Yan-An Wang. Department of Oral and Maxillofacial-Head and Neck Oncology, Ninth People's Hospital, School of \\ Medicine, Shanghai Jiao Tong University, 639 Zhizaoju Road, Shanghai 200011, China. Email: mingliu_jy@hotmail.com; wangyan_an@163.com.
}

Background: This study evaluated the levels, prevalence of anxiety and depression among patients with oral and maxillofacial venous malformations, along with associated factors.

Methods: Anxiety and depression, illness perceptions, and social support status of 69 patients with oral and maxillofacial venous malformations were analyzed using the Hospital Anxiety and Depression Scale, Brief Illness Perception Questionnaire, and Social Support Rating Scale, respectively. Eighty healthy controls were matched to the patients by sex, age, monthly income, education level, marital status, and employment status.

Results: Patients exhibited significantly higher levels of anxiety $(3.41 \pm 3.01$ vs. $1.03 \pm 1.66 ; \mathrm{P}<0.001)$ and depression $(7.14 \pm 2.47$ vs. 2.19 $\pm 2.12 ; \mathrm{P}<0.001)$ compared to controls. Eleven $(15.9 \%)$ and $30(43.5 \%)$ patients had clinical symptoms of anxiety and depression respectively, compared to $3.8 \%$ and $6.3 \%$ of the healthy controls, respectively. Thirty-three patients (47.8\%) had clinical symptoms of anxiety and/or depression, compared to $7.5 \%$ of the healthy controls. Multivariate analyses identified that facial lesions (odds ratio: 17.79, 95\% confidence interval: 1.22-259.66; $\mathrm{P}=0.035$ ), poor utility of social support (odds ratio: $0.02,95 \%$ confidence interval: $0.01-0.31 ; \mathrm{P}=0.006$ ), and poor emotional illness perception (odds ratio: $27.39,95 \%$ confidence interval: $5.01-149.89 ; \mathrm{P}<0.001)$ were significantly associated with anxiety and depression in patients.

Conclusions: Patients with oral and maxillofacial venous malformations displayed significantly increased levels and prevalence of anxiety and depression. These findings suggest the need for a standardized treatment for such patients, including appropriate medical intervention, psychological consultation, and social support.

Keywords: Anxiety; depression; social support; vascular malformations; oromaxillofacial (OMF)

Submitted Mar 16, 2021. Accepted for publication Jul 14, 2021.

doi: 10.21037/apm-21-608

View this article at: https://dx.doi.org/10.21037/apm-21-608

^ ORCID: Ming Liu, 0000-0003-3042-6603; Yan-An Wang, 0000-0001-7805-164X; Xiaochen Zhang, 0000-0002-5681-3444. 


\section{Introduction}

Venous malformations (VMs) represent the most common congenital vascular diseases, with a prevalence of $1 \%$ in the general population and an incidence of $1-5$ in 10,000 newborns $(1,2)$. VMs can be located anywhere in the body; however, approximately $40 \%$ occur in the head and neck, including the oromaxillofacial (OMF) area (3-5). Depending on the location and size, symptoms range from superficial asymptomatic varicosities to large deforming lesions accompanied by chronic or recurrent swelling and pain (5). As they are present at birth and grow proportional to the body without regress, VMs are challenging to treat $(6,7)$. However, VMs are usually not life-threatening and are mainly a cosmetic problem, particularly when they occur on visible parts of the body, such as the OMF region. Nevertheless, the impact of VM on a person's appearance may induce psychological distress among patients.

Psychological distress is multi-factorial and related to unpleasant emotional experiences, and it is common in patients with chronic physical disorders $(8,9)$. Anxiety and depression represent the most common symptoms of psychological distress, and they are associated with poor quality of life, an impaired ability to address disease, cognitive dysfunction, difficulty working, and social inhibition $(10,11)$. Moreover, individuals with symptoms of anxiety and depression have a higher morbidity and mortality risk from somatic diseases through low adherence to medical advice and treatment as compared to their healthier counterparts (12-14). Kessler et al. (9) found that anxiety and depression incur annual societal costs of tens of billions of dollars. However, these conditions are often treatable; thus, to improve treatment effectiveness and reduce the associated societal burden, early recognition of anxiety and depression is important for physicians, patients, and patients' family members (15). It is vital to investigate factors that are potentially correlated with anxiety and depression, as this can contribute to identifying patients who are more likely to develop these mental health issues. Sex, age, monthly income, education level, marital status, and employment status are significantly associated with anxiety or depression in the general population $(16,17)$. Further, those who are younger, a woman, unmarried, of a low education level, experiencing poor financial strain, and working low-paying jobs are more likely to show anxiety or depressive symptomatology as compared to their counterparts $(9,16,17)$.

Most studies concerning psychological distress focused on autoimmune systemic diseases, cardiovascular diseases, and cancers (18-20). Prior studies have reported that $13-17 \%$ of patients with congenital vascular malformations experience psychological distress (21), while $23.2 \%$ of patients with Klippel-Trenaunay syndrome are diagnosed with a mental illness (22). However, little is known about the severity of anxiety and depressive symptoms in patients with oral and maxillofacial VMs (OMFVMs) and, to our knowledge, no previous study has examined this topic. We thus assessed the severity of anxiety and depression symptoms in patients with OMFVMs to identify potentially correlated factors.

We present the following article in accordance with the STROBE reporting checklist (available at https://dx.doi. org/10.21037/apm-21-608).

\section{Methods}

This was a single institution, cross-sectional, observational study. All procedures were performed in accordance with established standards. The study was conducted in accordance with the Declaration of Helsinki (as revised in 2013). The study was approved by the ethical issues committee of the Ninth People's Hospital, affiliated with Shanghai Jiao Tong University School of Medicine, and informed consent was obtained from all individual participants.

\section{Patients}

Patients with OMFVMs ( $\mathrm{n}=69$ ) admitted to our clinic between December 2018 and December 2019 were recruited. The inclusion criteria were as follows: patients diagnosed with OMFVMs, aged $>18$ years, who can understand Chinese, and with normal cognitive functioning. Patients with a history of mental illness and/or with cognitive impairment were excluded from analyses. Sociodemographic characteristics and clinical data were collected via clinical interviews.

Additionally, a sample of healthy individuals $(\mathrm{n}=80)$ was recruited from the personnel working at the clinic and their relatives to serve as a healthy control (HC) group. HC participants were matched with the OMFVMs patients by sex, age, marital status, employment status, monthly income, and education level. Printed Chinese-language psychometric scales and questionnaires, including the Hospital Anxiety and Depression Scale (HADS), the Brief Illness Perception Questionnaire (B-IPQ), and the Social 
Support Rating Scale (SSRS) were completed in the clinic. All the Chinese versions of the scales and questionnaire used in this study have previously been validated $(10,23,24)$.

\section{Sociodemographic and clinical characteristics}

Sociodemographic (sex, age, monthly income, education level, marital status, and employment status) and clinical data (location and size of VMs) were collected through standardized interviews and assessments. The OMF area was divided into two parts: the facial and oral regions. The facial region was defined as the visible part containing the facial, nasal, temporal, orbital, and labial regions; the oral region comprised the buccal, lingual, palatal, oral floor, and labial mucosal regions. In accordance with the classification by Goyal et al. (25), the cut-off point was set at $5 \mathrm{~cm}$ to determine the size of lesions.

\section{Study scales and questionnaires}

\section{Assessment of anxiety and depression}

The Chinese version of the HADS was used to evaluate participants' symptoms of anxiety and depression. The HADS is a 14-item questionnaire that comprises two parts: seven items pertaining to anxiety and seven pertaining to depression. Each item is rated using a four-point scale (ranging from 0 to 3). For each subscale, possible scores range from 0 (no distress) to 21 (maximum distress). A score $>7$ for either subscale indicates clinically significant symptoms (10).

\section{Assessment of received social support}

The Chinese version of the SSRS was used to evaluate patients' social support status. This is a 10 -item questionnaire comprising three subscales: subjective support (provision of care, empathy, trust, and love), objective support (provision of help for patients in need), and utility of social support (actual utility of support received from social networks). Total scores range from 8 to 40 . Higher scores indicate reception of higher social support (26).

\section{Assessment of illness perceptions}

The Chinese version of the B-IPQ was used to assess the patients' illness perceptions. The B-IPQ questionnaire comprises eight items and evaluates three dimensions: cognitive representations of illness, emotional representations of illness, and illness comprehensibility. Five items assess cognitive representations of illness and relate to timeline, consequence, personal control, identity, and treatment control; two items assess emotional representations relating to concern and emotional response; and one item assesses comprehensibility of illness relating to understanding. Each item is rated using a 10-point scale (ranging from 1 to 10) (18).

\section{Statistical analysis}

Descriptive statistics were used to examine sociodemographic and clinical data. Data were presented as frequencies and percentages for categorical variables. The Shapiro-Wilk (W test) was conducted to determine the normality of continuous variables. Continuous variables were described as means \pm standard deviations when normally distributed or median (interquartile ranges) when not. Chi-square tests and $t$-tests or Wilcoxon non-parametric tests were conducted to identify significant differences between the patients with OMFVMs and the HC participants using two-tailed tests. Univariate and multivariate logistic regression analyses were conducted to identify potential predictors of anxiety or depression in the patients with OMFVMs. Variables that were significant $(\mathrm{P}<0.05)$ in the univariate analysis were entered the multivariate analysis. Statistical analyses were performed with SPSS 18.0 (SPSS Inc., Chicago, IL, USA). P values less than 0.05 were considered significant.

\section{Results}

Participants' sociodemographic characteristics and anxietyand depression-related variables are shown in Table 1. Patients' mean age was 38.5 years, ranging from 19 to 82 years. Most patients were women $(73.9 \%)$, married (68.1\%), and employed (62.3\%). Almost two-thirds (65.2\%) had a monthly income of less than 5,000 Chinese yuan, while only $24.6 \%$ had less than a high school education. Compared to the HC participants, patients' scores for anxiety $(3.41 \pm 3.01)$ and depression $(7.14 \pm 2.47)$ were significantly higher, indicating their increased levels of anxiety and depression. No significant differences were found between patients with OMFVMs and HCs on any other variables.

As shown in Table 2, according to the recommended criteria (using a cut-off point of $\geq 8$ ), $11(15.9 \%$ ) patients had anxiety and $30(43.5 \%)$ patients had depression. Overall, 33 (47.8\%) patients were identified with both anxiety and/or depressive symptoms. In contrast, among HC participants, only 3 (3.8\%) had anxiety, 5 (6.3\%) had depression, and $6(7.5 \%)$ had anxiety, depression, or both. 
Table 1 Sociodemographic characteristics and anxiety and depression scores of OMFVMs patients and HC participants

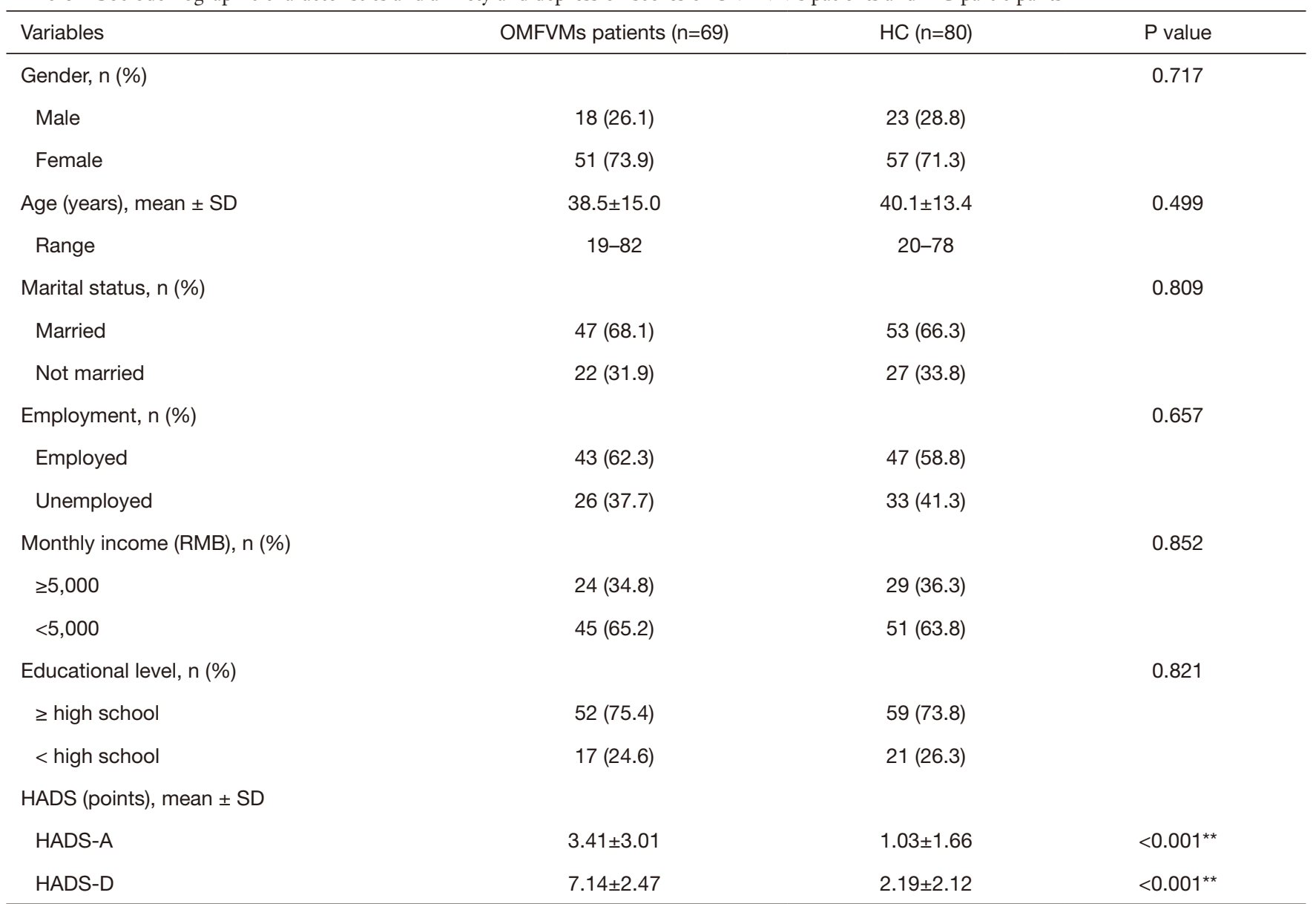

**, $\mathrm{P}<0.01$. OMFVMs, oral and maxillofacial venous malformations; HC, healthy control; HADS-A, hospital anxiety and depression scale-anxiety; HADS-D, hospital anxiety and depression scale-depression; SD, standard deviation.

Table 2 Prevalence of anxiety and depression in OMFVMs patients and HC participants

\begin{tabular}{lccc}
\hline Variables & OMFVMs patients $(n=69)$ & $H C(n=80)$ & $P$ value \\
\hline HADS-A & $11(15.9)$ & $3(3.8)$ & $0.011^{*}$ \\
HADS-D & $30(43.5)$ & $5(6.3)$ & $<0.001^{\star \star}$ \\
A and/or D & $33(47.8)$ & $6(7.5)$ & $<0.001^{\star \star}$ \\
\hline
\end{tabular}

*, $\mathrm{P}<0.05$; **, $\mathrm{P}<0.01$. OMFVMs, oral and maxillofacial venous malformations; HC, healthy control; HADS-A, hospital anxiety and depression scale-anxiety; HADS-D, hospital anxiety and depression scale-depression; A, anxiety; D, depression.

Thus, the prevalence of anxiety and depression among patients with OMFVMs was much higher than that among HC participants.

To investigate factors that are potentially associated with anxiety and depression, patients were divided into two groups: those with and those without anxiety and/ or depression. Univariate and multivariate logistic regression analyses revealed that sex, age, monthly income, education level, marital status, and employment status were non-significantly associated with patients' anxiety or depression (Table 3). Moreover, the presence of anxiety and/or depression among the patients was significantly associated with the location and diameter of their VMs (Table 3), the social support they received (Table 4), and 
Table 3 Sociodemographic and clinical characteristics of OMFVMs patients with anxiety and/or depression and without anxiety or depression

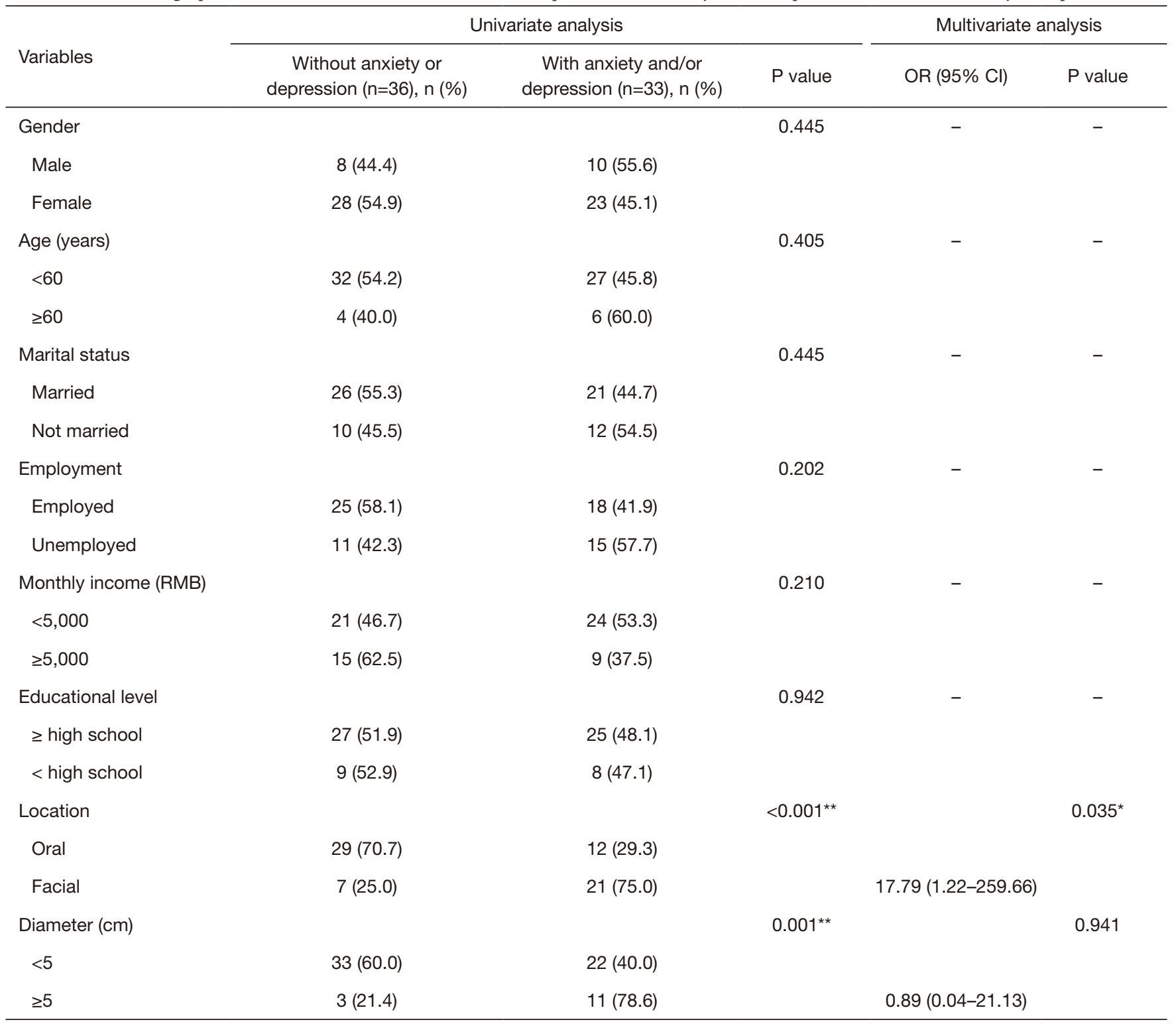

${ }^{*}, \mathrm{P}<0.05 ;{ }^{*}, \mathrm{P}<0.01$. OMFVMs, oral and maxillofacial venous malformations; OR, odds ratio; $\mathrm{Cl}$, confidence interval.

their illness perceptions (Table 5). The prevalence of anxiety and/or depression among patients with facial VMs was significantly higher than that in patients with oral VMs (75.0\% vs. $29.3 \%$, respectively; $\mathrm{P}<0.001)$. Similarly, significant differences were observed regarding lesion size, with the prevalence of anxiety and/or depression being higher among patients with larger lesions (diameter $\geq 5 \mathrm{~cm}$; 78.6\% vs. 40.0\%; $\mathrm{P}=0.010$; Table 3). Additionally, patients with anxiety and/or depression had less social support, including significantly lower subjective support (66.7\% vs.
23.3\%; $\mathrm{P}<0.001)$, lower objective support $(60.5 \%$ vs. $32.3 \%$; $\mathrm{P}=0.019)$, and lower utilization $(72.5 \%$ vs. $13.8 \% ; \mathrm{P}<0.001$; Table 4). Moreover, patients with anxiety and/or depression had worse illness perceptions; they had significantly higher scores for cognitive illness representations (67.7\% vs. 28.6\%; $\mathrm{P}=0.001)$, higher scores for emotional illness representations $(86.7 \%$ vs. $17.9 \% ; \mathrm{P}<0.001)$, and lower scores for illness comprehensibility $(61.7 \%$ vs. $18.2 \%$; $\mathrm{P}<0.001$; Table 5). The results of the multivariate analysis revealed significant correlations between facial VMs 
Table 4 Social support received by OMFVMs patients with anxiety and/or depression and without anxiety or depression

\begin{tabular}{|c|c|c|c|c|c|}
\hline Variables & \multicolumn{3}{|c|}{ Univariate analysis } & \multicolumn{2}{|c|}{ Multivariate analysis } \\
\hline Subjective $($ median score $=11$ ) & & & $<0.001^{\star \star}$ & & 0.734 \\
\hline$\leq 11$ & $13(33.3)$ & $26(66.7)$ & & $0.64(0.05-8.19)$ & \\
\hline$\leq 7$ & $15(39.5)$ & $23(60.5)$ & & $0.41(0.04-4.64)$ & \\
\hline$>7$ & $21(67.7)$ & $10(32.3)$ & & & \\
\hline Utilization (median score =8) & & & $<0.001^{\star \star}$ & & $0.006^{\star \star}$ \\
\hline
\end{tabular}

*, $\mathrm{P}<0.05 ;{ }^{* *}, \mathrm{P}<0.01$. OMFVMs, oral and maxillofacial venous malformations; OR, odds ratio; $\mathrm{Cl}$, confidence interval.

Table 5 Illness perception in OMFVMs patients with anxiety and/or depression and without anxiety or depression

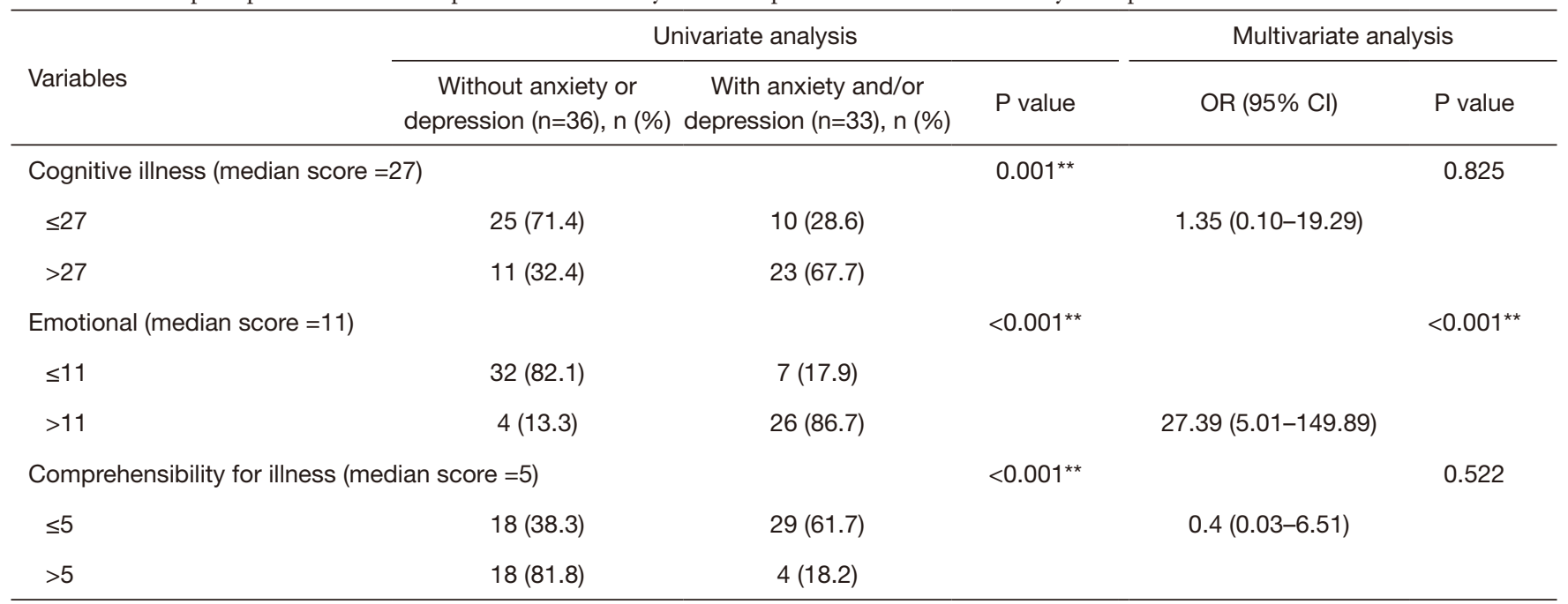

**, $\mathrm{P}<0.01$. OMFVMs, oral and maxillofacial venous malformations; OR, odds ratio; Cl, confidence interval.

[odds ratio (OR): 17.79; 95\% confidence interval (CI): 1.22-259.66; $\mathrm{P}=0.035$ ], lower utilization of available social support (OR: 0.02; 95\% CI: 0.01-0.31; $\mathrm{P}=0.006$ ), and poor emotional illness perception (OR: 27.39; 95\% CI: 5.01149.89; $\mathrm{P}<0.001)$ and anxiety and/or depression among patients with OMFVMs (Tables 3-5).

\section{Discussion}

VMs are very common in clinical practice. Moreover, they pose a challenge to OMF surgeons owing to the aesthetic and/or functional deficiencies they cause (especially VMs that occur in the OMF area). Further, curative treatment of VMs is rarely possible (27). Consequently, the main goal of treatment for patients with OMFVMs is to minimize functional impairment, aesthetic deficiency, and pain (28). Consequently, these lesions often remain as chronic diseases and may induce psychological distress and negatively impact quality of life. Furthermore, among patients with cancer, heart disease, autoimmune disease, and other chronic diseases, psychopathological symptoms such as anxiety and depression have important effects on illness progression 
through various pathways $(19,20,29)$. This novel study thus focused on anxiety and depression, and their predictive factors, among patients with OMFVMs.

Most patients with OMFVMs in our sample were women. This finding differed from previous findings, which indicated the absence of sex differences in this regard $(27,28)$. A possible reason for this discrepancy is that our sample was selected from individuals who voluntarily attended an $\mathrm{OMF}$ clinic, and female patients may be more likely to seek medical support than male patients. Patients exhibited increased levels of anxiety and depressive symptoms compared to HC participants. Moreover, the prevalence of the clinical symptoms of anxiety and depression among patients was significantly higher than that of HC participants. It could be assumed that the impact of $\mathrm{VMs}$ located in the OMF region on a person's appearance produces greater psychological burden owing to its conspicuous location. Moreover, chronic illness causes anxiety and depressive symptoms because of the prolonged disease course.

The prevalence of depression and/or anxiety among HCs $(7.5 \%)$, most of whom were women, was not higher than that of the general population (9). However, appearance issues relating to disease deformity may affect women more than men (30). This discrepancy could be explained by the fact that, with economic and societal development, men are also beginning to pay attention to their personal appearance. Thus, aesthetic deficiencies in visible parts of the body, such as the OMF region, would produce more psychological distress in men as compared with previous observations (30).

It is critical to investigate factors that are potentially correlated with anxiety and depression, as this can contribute to identifying patients who are more likely to develop these mental health issues and allow the implementation of preventive measures. Being younger, a woman, and having a low education level are correlated with anxiety or depressive symptomatology (31); however, other studies did not reveal any significant differences regarding psychological features $(32,33)$. In the present study, sex, age, monthly income, education level, marital status, employment status, and lesion diameter were non-significantly associated with anxiety or depression among patients with OMFVMs. However, facial VMs and poor social support and illness perceptions were significantly correlated with anxiety and/or depression among patients with OMFVMs. Further, patients with facial VMs were more likely to exhibit symptoms of anxiety or depression than patients with oral VMs, regardless of lesion diameter. This finding indicated that psychological distress is more dependent on lesion location than lesion size, especially when the lesions are located in regions that affect appearance. This might be because patients with more (vs. less) appearance defects have difficulty assessing their problems in a positive manner.

In addition, lower satisfaction with received social support was associated with anxiety and/or depression levels among patients. The utilization of social support among patients with OMFVMs with anxiety or depression was significantly lower than that of patients without anxiety or depression, which implies that the former group found the social support available in their environments to be less useful than did the latter group. This difference might be partially ascribed to lower extraversion among patients, which is characterized by a greater tendency toward selfcontrol or self-inhibition (34). Previous studies reported that anxiety and depression symptomatology is negatively correlated with levels of extraversion, and that extraversion predicts better psychological adjustment to illness, most likely through better utilization of social support $(34,35)$. Thus, since patients' relatives and colleagues are included in their social support network, they should be encouraged to provide help and advice more positively and actively to patients with OMFVMs.

Finally, patients with OMFVMs who had poor illness perceptions were more vulnerable to anxiety and depression symptoms than their counterparts. This was specifically found among patients with poor emotional perceptions of illness. These patients mentioned relatively more worries and negative perspectives regarding their illness over the preceding year, which resulted in more emotional distress.

The current findings provide novel information regarding anxiety and depression among patients with OMFVMs and can act as an essential resource that clinicians can use to help patients alleviate their symptoms. However, this study had several limitations. First, the number of patients with OMFVMs that were recruited was small, and all were recruited from a single institution. Since we attempted to investigate psychological distress, a larger sample sourced from multiple institutions would help generalize our conclusions. Second, patients were identified as having anxiety or depressive symptoms only according to the HADS criterion, which has been validated and is widely used to measure psychological distress. However, since psychological distress is widely dispersed across the general population, conclusions should be drawn with caution. In future studies, psychiatrists should examine the data. Third, this was a cross-sectional observational study. Future studies should investigate whether improvements in facial lesions, 
social support, and illness perception can improve patients' symptoms of anxiety and/or depression over time. Lastly, we did not assess patients' personality characteristics.

\section{Conclusions}

Patients with OMFVMs displayed significantly higher anxiety and depressive symptomatology compared to HC participants. Anxiety and depressive symptoms in patients with OMFVMs were significantly associated with facial VMs and poor utility of social support and emotional illness perception. The above results underline the necessity of providing these patients with appropriate medical care, psychological consultation, and social support. Our findings suggest the need for a standardized comprehensive treatment for patients with OMFVMs.

\section{Acknowledgments}

The authors express their appreciation to Dr. Xiao Zhang for assistance regarding statistical interpretation of this work. Funding: This work was supported by the National Natural Science Foundation of China (81801023).

\section{Footnote}

Reporting Checklist: The authors have completed the STROBE reporting checklist. Available at https://dx.doi. org/10.21037/apm-21-608

Data Sharing Statement: Available at https://dx.doi. org/10.21037/apm-21-608

Conflicts of Interest: All authors have completed the ICMJE uniform disclosure form (available at https://dx.doi. org/10.21037/apm-21-608). The authors have no conflicts of interest to declare.

Ethical Statement: The authors are accountable for all aspects of the work in ensuring that questions related to the accuracy or integrity of any part of the work are appropriately investigated and resolved. The study was conducted in accordance with the Declaration of Helsinki (as revised in 2013). The study was approved by the ethical issues committee of the Ninth People's Hospital, affiliated with Shanghai Jiao Tong University School of Medicine, and informed consent was obtained from all individual participants. All authors confirm that all the tables in the manuscript are original.

Open Access Statement: This is an Open Access article distributed in accordance with the Creative Commons Attribution-NonCommercial-NoDerivs 4.0 International License (CC BY-NC-ND 4.0), which permits the noncommercial replication and distribution of the article with the strict proviso that no changes or edits are made and the original work is properly cited (including links to both the formal publication through the relevant DOI and the license). See: https://creativecommons.org/licenses/by-nc-nd/4.0/.

\section{References}

1. Legiehn GM, Heran MK. Venous malformations: classification, development, diagnosis, and interventional radiologic management. Radiol Clin North Am 2008;46:545-97, vi.

2. Vikkula M, Boon LM, Mulliken JB. Molecular genetics of vascular malformations. Matrix Biol 2001;20:327-35.

3. Behravesh S, Yakes W, Gupta N, et al. Venous malformations: clinical diagnosis and treatment. Cardiovasc Diagn Ther 2016;6:557-69.

4. Cahill AM, Nijs EL. Pediatric vascular malformations: pathophysiology, diagnosis, and the role of interventional radiology. Cardiovasc Intervent Radiol 2011;34:691-704.

5. Seront E, Vikkula M, Boon LM. Venous Malformations of the Head and Neck. Otolaryngol Clin North Am 2018;51:173-84.

6. Ali S, Mitchell SE. Outcomes of Venous Malformation Sclerotherapy: A Review of Study Methodology and LongTerm Results. Semin Intervent Radiol 2017;34:288-93.

7. van der Vleuten CJ, Kater A, Wijnen MH, et al. Effectiveness of sclerotherapy, surgery, and laser therapy in patients with venous malformations: a systematic review. Cardiovasc Intervent Radiol 2014;37:977-89.

8. Bultz BD, Carlson LE. Emotional distress: the sixth vital sign in cancer care. J Clin Oncol 2005;23:6440-1.

9. Kessler RC, Aguilar-Gaxiola S, Alonso J, et al. The global burden of mental disorders: an update from the WHO World Mental Health (WMH) surveys. Epidemiol Psichiatr Soc 2009;18:23-33.

10. Li Q, Lin Y, Hu C, et al. The Chinese version of hospital anxiety and depression scale: Psychometric properties in Chinese cancer patients and their family caregivers. Eur J Oncol Nurs 2016;25:16-23.

11. Steel JL, Geller DA, Gamblin TC, et al. Depression, immunity, and survival in patients with hepatobiliary carcinoma. J Clin Oncol 2007;25:2397-405. 
12. Penninx BW, Milaneschi Y, Lamers F, et al. Understanding the somatic consequences of depression: biological mechanisms and the role of depression symptom profile. BMC Med 2013;11:129.

13. Williams JB, Alexander KP, Morin JF, et al. Preoperative anxiety as a predictor of mortality and major morbidity in patients aged $>70$ years undergoing cardiac surgery. Am J Cardiol 2013;111:137-42.

14. Ojard C, Donnelly JP, Safford MM, et al. Psychosocial stress as a risk factor for sepsis: a population-based cohort study. Psychosom Med 2015;77:93-100.

15. Boeschoten RE, Braamse AMJ, Beekman ATF, et al. Prevalence of depression and anxiety in Multiple Sclerosis: A systematic review and meta-analysis. J Neurol Sci 2017;372:331-41.

16. Almeida OP, Draper B, Pirkis J, et al. Anxiety, depression, and comorbid anxiety and depression: risk factors and outcome over two years. Int Psychogeriatr 2012;24:1622-32.

17. Askim Å, Gustad LT, Paulsen J, et al. Anxiety and Depression Symptoms in a General Population and Future Risk of Bloodstream Infection: The HUNT Study. Psychosom Med 2018;80:673-9.

18. Chung J, Ju G, Yang J, et al. Prevalence of and factors associated with anxiety and depression in Korean patients with newly diagnosed advanced gastrointestinal cancer. Korean J Intern Med 2018;33:585-94.

19. Denollet J, Sys SU, Stroobant N, et al. Personality as independent predictor of long-term mortality in patients with coronary heart disease. Lancet 1996;347:417-21.

20. Golemati CV, Moutsopoulos HM, Vlachoyiannopoulos PG. Psychological characteristics of systemic sclerosis patients and their correlation with major organ involvement and disease activity. Clin Exp Rheumatol 2013;31:37-45.

21. Fahrni JO, Cho EY, Engelberger RP, et al. Quality of life in patients with congenital vascular malformations. J Vasc Surg Venous Lymphat Disord 2014;2:46-51.

22. Harvey JA, Nguyen H, Anderson KR, et al. Pain, psychiatric comorbidities, and psychosocial stressors associated with Klippel-Trenaunay syndrome. J Am Acad Dermatol 2018;79:899-903.

23. Chen P, Broadbent E, Coomarasamy C, et al. Illness perception in association with psychological functioning in patients with discoid lupus erythematosus. Br J Dermatol 2015;173:824-6.

24. Yu J, Bang KS. Perceived Alienation of, and Social Support for, Siblings of Children With Cancer. J Pediatr Oncol Nurs 2015;32:410-6.
25. Goyal M, Causer PA, Armstrong D. Venous vascular malformations in pediatric patients: comparison of results of alcohol sclerotherapy with proposed MR imaging classification. Radiology 2002;223:639-44.

26. Li J, Wu X, Lin J, et al. Type D personality, illness perception, social support and quality of life in continuous ambulatory peritoneal dialysis patients. Psychol Health Med 2017;22:196-204.

27. Dompmartin A, Vikkula M, Boon LM. Venous malformation: update on aetiopathogenesis, diagnosis and management. Phlebology 2010;25:224-35.

28. Hage AN, Chick JFB, Srinivasa RN, et al. Treatment of Venous Malformations: The Data, Where We Are, and How It Is Done. Tech Vasc Interv Radiol 2018;21:45-54.

29. Reiche EM, Nunes SO, Morimoto HK. Stress, depression, the immune system, and cancer. Lancet Oncol 2004;5:617-25.

30. van Lankveld WG, Vonk MC, Teunissen H, et al. Appearance self-esteem in systemic sclerosis--subjective experience of skin deformity and its relationship with physician-assessed skin involvement, disease status and psychological variables. Rheumatology (Oxford) 2007;46:872-6.

31. Moser DK, Clements PJ, Brecht ML, et al. Predictors of psychosocial adjustment in systemic sclerosis. The influence of formal education level, functional ability, hardiness, uncertainty, and social support. Arthritis Rheum 1993;36:1398-405.

32. Hyphantis TN, Tsifetaki N, Pappa C, et al. Clinical features and personality traits associated with psychological distress in systemic sclerosis patients. J Psychosom Res 2007;62:47-56.

33. Nguyen C, Bérezné A, Baubet T, et al. Association of gender with clinical expression, quality of life, disability, and depression and anxiety in patients with systemic sclerosis. PLoS One 2011;6:e17551.

34. Carver CS, Connor-Smith J. Personality and coping. Annu Rev Psychol 2010;61:679-704.

35. Segerstrom SC. Individual differences, immunity, and cancer: lessons from personality psychology. Brain Behav Immun 2003;17 Suppl 1:S92-7.

Cite this article as: Zhang $\mathrm{X}$, Zhao $\mathrm{X}$, Wu P, Zhao Z, Liu M, Wang YA. Prevalence and associated factors of anxiety and depression among patients with oromaxillofacial venous malformations. Ann Palliat Med 2021;10(8):8642-8650. doi: 10.21037/apm-21-608 